\title{
Physical properties and H-ionizing-photon production rates of extreme nearby star-forming regions
}

\author{
Jacopo Chevallard, ${ }^{1 \star}, \dagger$ Stéphane Charlot,${ }^{2}$ Peter Senchyna, ${ }^{3}$ Daniel P. Stark, ${ }^{3}$ \\ Alba Vidal-García, ${ }^{2}$ Anna Feltre, ${ }^{4}$ Julia Gutkin, ${ }^{2}$ Tucker Jones, ${ }^{5,6} \ddagger$ Ramesh Mainali ${ }^{3}$ \\ and Aida Wofford ${ }^{7}$ \\ ${ }^{1}$ Scientific Support Office, Directorate of Science and Robotic Exploration, ESA/ESTEC, Keplerlaan 1, NL-2201 AZ Noordwijk, the Netherlands \\ ${ }^{2}$ Sorbonne Universités, UPMC-CNRS, UMR7095, Institut d'Astrophysique de Paris, F-75014 Paris, France \\ ${ }^{3}$ Steward Observatory, University of Arizona, 933 N Cherry Ave, Tucson, AZ 85721 USA \\ ${ }^{4}$ Centre de Recherche Astrophysique de Lyon, Université Lyon 1, 9 Avenue Charles André, F-69561 Saint Genis Laval Cedex, France \\ ${ }^{5}$ Department of Physics, University of California Davis, 1 Shields Avenue, Davis, CA 95616, USA \\ ${ }^{6}$ Institute for Astronomy, University of Hawaii, 2680 Woodlawn Drive, Honolulu, HI 96822, USA \\ ${ }^{7}$ Instituto de Astronomía, UNAM, Ensenada, CP 22860 Baja California, Mexico
}

Accepted 2018 June 1. Received 2018 June 1; in original form 2017 September 12

\begin{abstract}
Measurements of the galaxy UV luminosity function at $z \gtrsim 6$ suggest that young stars hosted in low-mass star-forming galaxies produced the bulk of hydrogen-ionizing photons necessary to reionize the intergalactic medium (IGM) by redshift $z \sim 6$. Whether star-forming galaxies dominated cosmic reionization, however, also depends on their stellar populations and interstellar medium properties, which set, among other things, the production rate of $\mathrm{H}$-ionizing photons, $\xi_{\text {ion }}^{\star}$, and the fraction of these escaping into the IGM. Given the difficulty of constraining with existing observatories the physical properties of $z \gtrsim 6$ galaxies, in this work we focus on a sample of 10 nearby objects showing UV spectral features comparable to those observed at $z \gtrsim 6$. We use the new-generation BEAGLE tool to model the UV-to-optical photometry and UV/optical emission lines of these local 'analogues' of high-redshift galaxies, finding that our relatively simple, yet fully self-consistent, physical model can successfully reproduce the different observables considered. Our galaxies span a broad range of metallicities and are characterized by high ionization parameters, low dust attenuation, and very young stellar populations. Through our analysis, we derive a novel diagnostic of the production rate of $\mathrm{H}$-ionizing photons per unit UV luminosity, $\xi_{\text {ion }}^{\star}$, based on the equivalent width of the bright [О III] $\lambda \lambda 4959,5007$ line doublet, which does not require measurements of H-recombination lines. This new diagnostic can be used to estimate $\xi_{\text {ion }}^{\star}$ from future direct measurements of the [O III] $\lambda \lambda 4959,5007$ line using JWST/NIRSpec (out to $z \sim 9.5$ ), and by exploiting the contamination by $\mathrm{H}, \beta+[\mathrm{O} \mathrm{III}] \lambda \lambda 4959,5007$ of photometric observations of distant galaxies, for instance from existing Spitzer/IRAC data and from future ones with JWST/NIRCam.
\end{abstract}

Key words: methods: data analysis-H II regions-galaxies: dwarf-galaxies: evolutiongalaxies: ISM-dark ages, reionization, first stars .

\section{INTRODUCTION}

The appearance of the first stars and galaxies in the Universe at the end of the Dark Ages marked the beginning of the epoch of reionization (EoR, e.g. Bromm \& Yoshida 2011). The EoR ap-

*E-mail: chevallard@iap.fr

$\dagger$ ESA Research Fellow.

$\ddagger$ Hubble Fellow. pears to have lasted mostly between $z \sim 15$ (Planck Collaboration XLVII 2016) and $z \sim 6$ (e.g. Fan et al. 2006). Reionization is generally thought to have been mainly driven by young stars in low-mass galaxies (see review by Stark 2016), with minor contributions by active galactic nuclei (AGN) (e.g. Parsa, Dunlop $\&$ McLure 2017), X-ray binaries (e.g. Mirabel et al. 2011) and Population III stars (e.g. Wise et al. 2014). The observed steepening of the faint-end slope of the ultraviolet (UV) galaxy luminosity function at redshift $z \sim 4-8$ (e.g. Bouwens et al. 2015a; Finkelstein et al. 2015) in fact suggests that low-mass galaxies were 
more abundant in the early Universe than at more recent times, potentially providing enough $\mathrm{H}$-ionizing photons to reionize the IGM (e.g. Robertson et al. 2015; Bouwens et al. 2015b). However, this interpretation is subject to major uncertainties: current data do not allow any accurate determination of the abundances of lowmass galaxies during the EoR, because of degeneracies among the luminosity function parameters (e.g. Finkelstein et al. 2015) and the difficulty of probing magnitudes fainter than $M_{\mathrm{UV}} \sim-17$ (except in lensed fields, e.g. Atek et al. 2015a,b; Ishigaki et al. 2018). Another major source of uncertainty is the relation between the (non-ionizing) UV-continuum emission of EoR galaxies, their production rate of H-ionizing photons, $\dot{N}_{\text {ion }}$, and the number of these photons escaping from the galaxies, $f_{\text {esc }}$. Direct measurements of the rate of ionizing photons escaping from galaxies (the product $f_{\text {esc }} \times \dot{N}_{\text {ion }}$ ) are only possible at lower redshifts (e.g. Izotov et al. 2016; Vanzella et al. 2016), since any H-ionizing photon emitted by EoR galaxies would be absorbed by neutral $\mathrm{H}$ along the line of sight. Indirect diagnostics on $f_{\text {esc }}$ have been proposed, for instance based on the relation between $\mathrm{H}$-Balmer lines and UV-continuum emission (Zackrisson, Inoue \& Jensen 2013) and on the Ly $\alpha$-line profile (Verhamme et al. 2015, 2017), but have not yet been tested on EoR galaxies.

In this paper, we focus on the production rate of $\mathrm{H}$-ionizing photons per unit intrinsic monochromatic UV (1500 ̊) luminosity, $\xi_{\text {ion }}^{\star}=\dot{N}_{\text {ion }} / L_{\text {UV }}^{\star}$. This quantity depends only on the properties of the stellar populations emitting the ionizing photons, i.e. mainly age and metallicity. Analytical reionization models often assume a fixed value for $\xi_{\text {ion }}^{\star}$, typically in the range $\log \left(\xi_{\text {ion }}^{\star} / \mathrm{erg}^{-1} \mathrm{~Hz}\right) \sim$ 25.2-25.3 (e.g. Kuhlen \& Faucher-Giguère 2012; Robertson et al. 2013), although the dependence of $\xi_{\text {ion }}^{\star}$ on stellar population properties would introduce galaxy-to-galaxy variations (both stochastic and systematic). Here, we use the new-generation spectral analysis tool BEAGLE (Chevallard \& Charlot 2016) to measure the production rate of $\mathrm{H}$-ionizing photons of 10 extreme nearby star-forming regions found by Senchyna et al. (2017; hereafter S17) to have UV spectra similar to those of $z \sim 6-7$ star-forming galaxies. Our simultaneous modelling of several UV and optical emission lines also provide valuable constraints on the properties of the ionizing stars, photoionized gas, and attenuation by dust. Beyond the demonstration that the stellar population and photoionization models incorporated into BEAGLE offer accurate fits to the data, this analysis allows us to uncover and calibrate a relation between $\xi_{\text {ion }}^{\star}$ and equivalent width of $(\mathrm{H}, \beta+)[\mathrm{O}$ III $] \lambda \lambda 4959,5007$. This relation extends from canonical $\xi_{\text {ion }}^{\star}$ values at low line equivalent widths up to nearly an order or magnitude larger for the most extreme galaxies.

\section{DATA AND MODELLING APPROACH}

\subsection{Spectro-photometric observations of local 'analogues' of high-redshift galaxies}

We appeal to a sample of 10 nearby analogues of primeval galaxies, for which our team has acquired high-quality UV data from $H S T / C O S$ (G160M and G185M gratings) and optical spectra from MMT (see S17 for details). These galaxies were selected from a sample of He II $\lambda 4686$ emitters dominated by stellar photoionization, identified by Shirazi \& Brinchmann (2012) from the SDSS DR7 (Abazajian et al. 2009). These objects show extremely large equivalent widths of optical emission lines (e.g. $[\mathrm{O}$ III] $\lambda \lambda 4959,5007 \gtrsim 1000 \AA$ ), which, although rare in the nearby
Universe (only $\sim 0.01$ per cent of SDSS galaxies), they become far more common at higher redshifts (e.g. Smit et al. 2014; Rasappu et al. 2016). The 10 galaxies in our sample have hard ionizing spectra able to doubly ionize He, i.e. with substantial flux at $\lambda<228 \AA(54.4 \mathrm{eV})$, although only half show WolfRayet (WR) spectral features. Our follow-up HST observations of these objects succeeded in revealing high-ionization UV lines (C IV $\left.\left.\left.\lambda \lambda 1548,1551,\left[\mathrm{C}_{\text {III }}\right] \lambda 1907+\mathrm{C}_{\mathrm{III}}\right] \lambda 1909, \mathrm{O}_{\mathrm{III}}\right] \lambda \lambda 1660,1666\right)$ similar to those typically observed in galaxies at $z \gtrsim 5$ (Stark et al. 2015a,b, 2017; Mainali et al. 2017; Schmidt et al. 2017; Smit et al. 2017), providing a unique reference sample for high-redshift studies.

The location of our galaxies in the '[N II]' Baldwin, Phillips \& Terlevich (1981, BPT) diagram shown in Fig. 1(a) is typical of sub-solar metallicity gas $\left(Z / Z_{\odot} \sim 0.1-0.7\right)$ with a high ionization parameter (see, e.g. fig. 2 of Gutkin, Charlot \& Bruzual 2016). The large O32 values at fixed R23, shown in Fig. 1(b), also indicate a high ionization parameter, although these values can also be caused by densitybounded H II regions (e.g. Jaskot \& Oey 2013; Stasińska et al. 2015). As we show below, ionization-bounded regions are favoured by our model, which well matches the observed [O I] $\lambda 6363$ fluxes, which should appear weaker for density-bounded regions. Fig. 1(a) also shows that our galaxies have lower $\left[\mathrm{N}_{\text {II }}\right] \lambda 6584 / \mathrm{H}, \alpha$, at fixed $\left[\mathrm{O}_{\mathrm{III}}\right] \lambda 4959 / \mathrm{H}, \beta$, than those in the KBSS sample of Strom et al. (2017). These ratios are similar to those of the $z \sim 0.3$ LyC leakers of Izotov et al. (2016), although they do not reach the extreme [N II] $\lambda 6584 / \mathrm{H}, \alpha \sim-2.5$ of the compact star-forming galaxies of Izotov, Thuan \& Guseva (2017b). Interestingly, Fig. 1(b) shows no significant offset between our galaxies, the KBSS sample and the LyC leakers in the O32 versus R23 diagram, while Izotov et al. (2017b) specifically targeted extreme $\mathrm{O} 32$ emitters. We also note the presence of a broad $\left(\mathrm{a}\right.$ few $\left.\times 10^{2} \mathrm{~km} \mathrm{~s}^{-1}\right)$ component in several emission lines $([\mathrm{O} \mathrm{III}] \lambda \lambda 4959,5007, \mathrm{H} \beta, \mathrm{H} \alpha)$, at a level of a few per cent the intensity of the narrow component. Such lowintensity broad components have been observed in other low-mass, low-metallicity, highly star-forming galaxies and linked to energy injection from stellar winds and supernovae (e.g. Izotov, Thuan \& Guseva 2007; James et al. 2009; Amorín et al. 2012), since AGNs typically produce broader components $\left(\gtrsim 10^{3} \mathrm{~km} \mathrm{~s}^{-1}\right)$ with intensities comparable to the narrow ones (e.g. Izotov \& Thuan 2008; Izotov et al. 2010).

\subsection{Emission line fluxes measurements}

We measure the fluxes of the emission lines listed in Table 1 and their associated errors with a custom fitting software described in S17. In summary, for each line, or group of neighbouring lines, we consider a linear function to describe the local continuum and fit-the-line profile with a Gaussian function. For lines with multiple kinematic components, we consider a second Gaussian function. We do not attempt to subtract the underlying stellar absorptions from the measured line fluxes, since stellar absorptions are self-consistently included in our stellar population + photoionization model (see Section 2.3 below). We rescale the formal errors on the line fluxes measured from sDSs spectra by a factor 1.8, the average error scaling suggested by the MPA/JHU analysis. ${ }^{1}$ As in S17, we correct the line fluxes for Galactic extinction using the dust map of Schlafly \& Finkbeiner (2011) and assuming the $R_{V}=3.1$ extinction curve of Fitzpatrick (1999). Since the predictions of our model include only

\footnotetext{
${ }^{1}$ https://wwwmpa.mpa-garching.mpg.de/SDSS/DR7/raw_data.html
} 

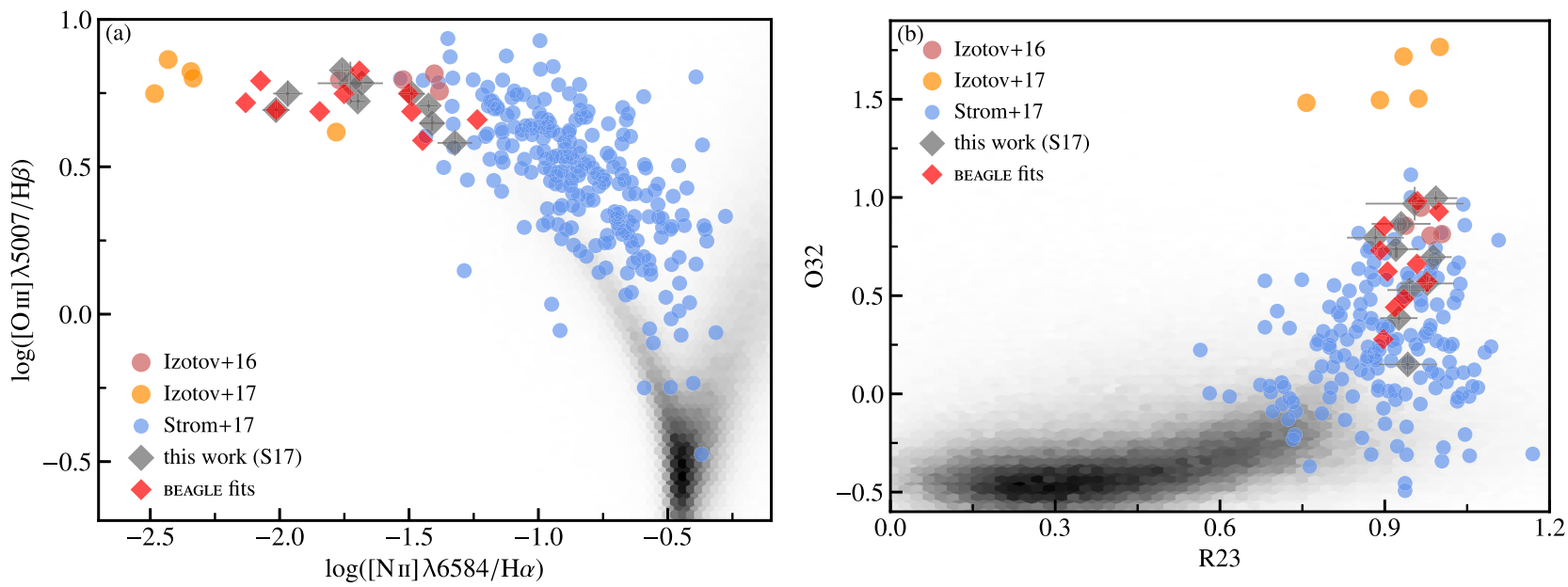

Figure 1. (a) [N II] BPT diagram showing star-forming galaxies at $z \sim 2-2.6$ from the KBSS survey (blue circles, Strom et al. 2017), the 4 LyC continuum 'leaker' at $z \sim 0.3$ from Izotov et al. (2016) (red circles), the five compact star-forming galaxies at $z<0.07$, candidates LyC continuum leakers, from Izotov et al. (2017b), and the 10 galaxies presented in S17 analysed in this work (grey diamonds), along with the model predictions obtained with BEAGLE (red diamonds). As a reference, we also show with a grey-scale (linear) density plot galaxies from the MPA/JHU emission line catalogue based on sDss DR7 data. MPA/JHU fluxes are corrected for Galactic extinction only, while the other fluxes for internal extinction as well. (b) same as (a), but showing the $\mathrm{O} 32=\log ([\mathrm{O}$ III $] \lambda \lambda 4959,5007 /[\mathrm{O}$ II $] \lambda \lambda 3726,3729)$ versus $\mathrm{R} 23=\log \left([\mathrm{O}\right.$ III $\left.\left.] \lambda \lambda 4959,5007+\left[\mathrm{O}_{\text {II }}\right] \lambda \lambda 3726,3729\right) / \mathrm{H}, \beta\right)$ diagram.

H-Balmer transitions of principal quantum number $n \leq 6$, where $n$ $=6$ corresponds to $\mathrm{H} \delta$, we subtract from the measured $\mathrm{He}_{\mathrm{I}} \lambda 3889$ flux the contribution from the $\mathrm{H} \zeta \lambda 3889$ line using the measured $\mathrm{H} \delta$ flux, and assuming a case B recombination ratio of $\mathrm{H} \zeta / \mathrm{H} \delta=0.41$ valid for a $T=10^{4} \mathrm{~K}$ gas (see table 4.2 of Osterbrock \& Ferland 2006). ${ }^{2}$ Given the similar (circular) apertures of sDSs fibers and $H S T / C O S$ (diameters of 3 and 2.5 arcsec, respectively), we do not apply any aperture correction to the UV and optical emission lines measurements.

\subsection{Stellar-population and photoionization modelling}

We model the UV-to-optical photometry and emission lines integrated fluxes of our galaxies to constrain their physical properties and production rate of $\mathrm{H}$-ionizing photons. To achieve this, we use BEAGLE, a new-generation spectral analyses tool which incorporates a self-consistent description of the emission from stars (based on the latest version of the Bruzual \& Charlot 2003 stellar population synthesis $\operatorname{code}^{3}$ ) and photoionized gas (using CLOUDY version 13.3, last described in Ferland et al. 2013). In practice, we consider a Chabrier (2003) stellar initial mass function truncated at 0.1 and $100 \mathrm{M}_{\odot}$, and adopt a constant star formation rate and a constant metallicity. The star formation and chemical enrichment history of our galaxies is hence parametrized in terms of the galaxy stellar mass $M$, age of the oldest stars $t$ (or age of the galaxy) and stellar metallicity $Z$. Following S17, we account for the effect of dust attenuation by considering the Small Magellanic Cloud extinction curve (Pei 1992), and vary the $V$-band attenuation optical depth $\hat{\tau}_{V}$. The choice of using an extinction curve is justified by the fact that

\footnotetext{
${ }^{2}$ The proximity in wavelength of $\mathrm{H} \delta$ at $\lambda=4101.7 \AA$ to $\mathrm{H} \zeta \lambda 3889$ allows us to ignore the potential impact of dust attenuation on the estimation of the $\mathrm{H} \zeta$ flux, since $\mathrm{H} \delta$ and $\mathrm{H} \zeta$ would suffer a comparable amount of attenuation. ${ }^{3}$ See appendix A of Vidal-García et al. 2017 for a comparison of the population synthesis code used in this work with the original version of Bruzual \& Charlot (2003).
}

geometry and scattering effects should have only a minor influence on line emission from the small physical regions probed by our data.

We follow Gutkin et al. (2016) to account for (line + continuum) nebular emission. We fix the gas density at $n_{\mathrm{H}}=10^{2} \mathrm{~cm}^{-3}$, the typical value observed in local compact star-forming galaxies (e.g. Izotov et al. 2006; Jaskot \& Oey 2013), set the interstellar metallicity equal to the stellar metallicity $\left(Z_{\text {ISM }}=Z\right)$, and vary the ionization parameter $\log U_{\mathrm{S}}$, which sets the ratio of $\mathrm{H}$-ionizing-photon to gas density at the Strömgren radius ${ }^{4}$ the dust-to-metal mass ratio $\xi_{\mathrm{d}}$, which sets the depletion of heavy elements onto dust grains, and the carbon-to-oxygen abundance ratio $\mathrm{C} / \mathrm{O}$, where, $\mathrm{C} / \mathrm{O}=1$ implies a scaled-Solar abundance.

We perform two separate fittings with BEAGLE: one to a combination of UV and optical photometry and Balmer emission lines, to derive the intrinsic UV luminosity of our galaxies; the other to a broad set of UV and optical emission lines, to derive the physical properties of the ionized gas and ionization sources. In the combined photometry + Balmer lines fit, we consider the $\mathrm{H}$-Balmer lines $\mathrm{H} \alpha$, $\mathrm{H} \beta, \mathrm{H} \gamma$, and $\mathrm{H} \delta$, the ugri optical (fiber) photometry from sDSs, ${ }^{5}$ and a single UV photometric band, which we compute from an integration of the HST/COS spectra. ${ }^{6}$ Because of the different filter configurations used when acquiring the COS data, we consider a flat filter covering the region $\lambda \sim 1620-1660 \AA$ for the objects SB 36 and SB 182, and a filter covering the range $\lambda \sim 1480-1515 \AA$ for the other eight objects. Both regions sample the rest-frame UV continuum of the galaxies, and are not significantly affected by nebular emission lines or ISM absorption lines. Since Balmer lines alone cannot constrain the several nebular emission parameters,

\footnotetext{
${ }^{4}$ This parametrization translates into a volume-averaged ionization parameter $\langle U\rangle=9 / 4 U_{\mathrm{S}}$ (see equation 1 of Hirschmann et al. 2017).

${ }^{5} \mathrm{We}$ do not fit the reddest SDSs band $(z)$ since the adopted (constant) star formation histories may introduce biases when fitting a band potentially dominated by evolved stellar populations.

${ }^{6}$ Note that we do not use GALEX photometry, to avoid potential biases introduced by the large GALEX PSF.
} 
Table 1. UV and optical emission lines fluxes computed from the HST/COS, MMT, and SDSS spectra, along with the fluxes predicted by our model. The model fluxes correspond to the posterior median of the marginal posterior distribution of each line flux. Although in our analysis we fitted the individual flux measurements, in this table, to facilitate the intepretation, we express the fluxes as $\log [F / F(\mathrm{H}, \alpha)]$, where $F$ is the flux of any emission line and $F(\mathrm{H}, \alpha)$ is the observed $\mathrm{H} \alpha$ flux. The complete table is available online.

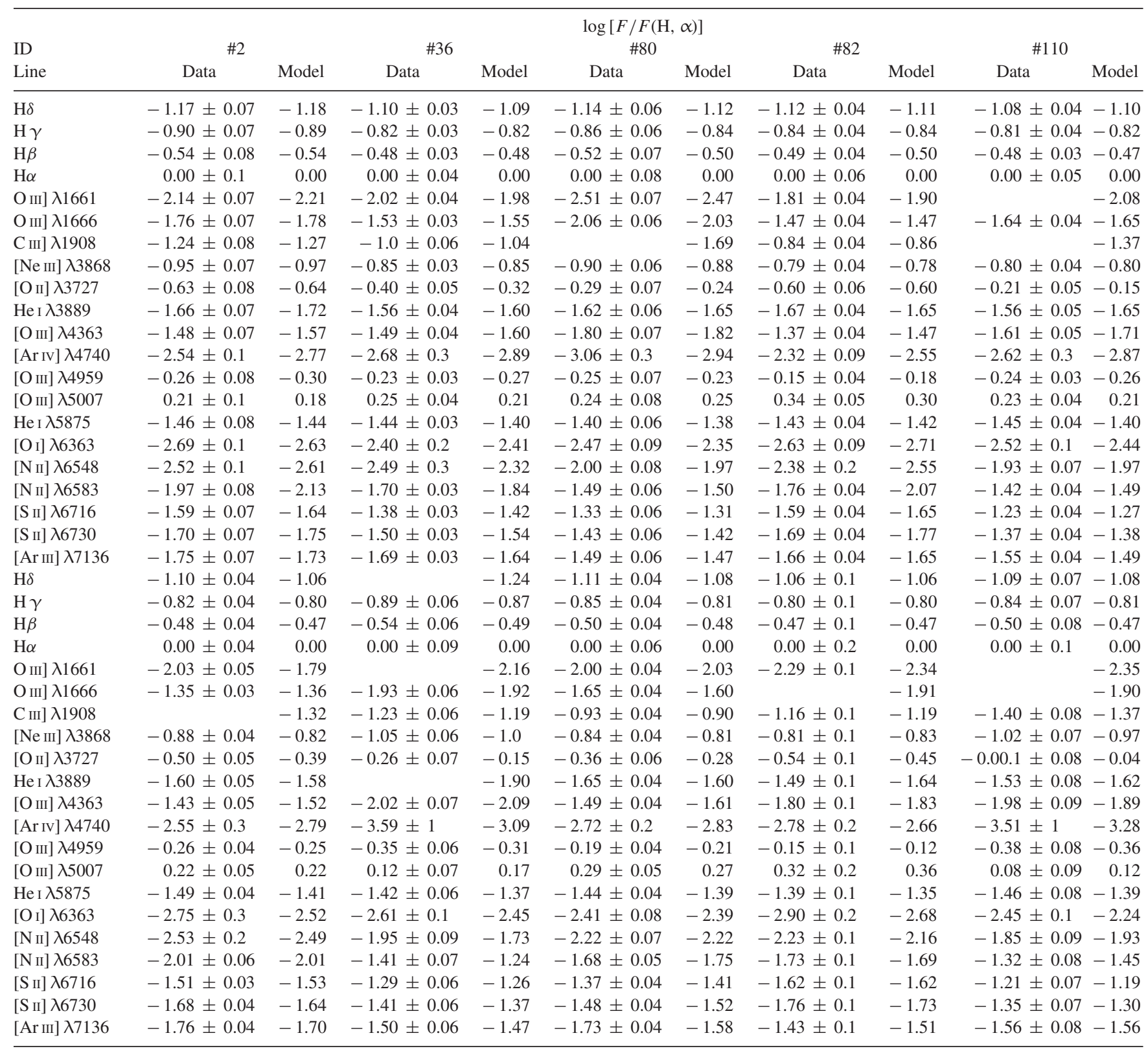

we simplify the model and fix the carbon-to-oxygen abundance ratio to the Solar value $\left[\mathrm{C} / \mathrm{O}=(\mathrm{C} / \mathrm{O})_{\odot}\right]$ and the depletion factor to $\xi_{\mathrm{d}}=0.3$, to obtain a model described by the five free parameters $\boldsymbol{\Theta}^{\text {phot+Balmer }}=\left[\log \left(M / \mathrm{M}_{\odot}\right), \log (t / \mathrm{yr}), \log \left(Z / \mathrm{Z}_{\odot}\right), \log U_{\mathrm{S}}, \hat{\tau}_{V}\right]$.

In a separate fitting, we model the broad range of UV and optical emission lines listed in Table 1. Besides the H-Balmer lines $\mathrm{H} \alpha, \mathrm{H} \beta$, $\mathrm{H} \gamma$, and $\mathrm{H} \delta$, we include several $\mathrm{O}, \mathrm{O}^{+}$, and $\mathrm{O}^{2+}$ lines, the nitrogen and sulphur doublets $\left[\mathrm{N}_{\mathrm{II}}\right] \lambda \lambda 6548,6584$ and $\left[\mathrm{S}_{\mathrm{II}}\right] \lambda \lambda 6716,6731$, the $\left.\left[\mathrm{C}_{\mathrm{III}}\right] \lambda 1907+\mathrm{C}_{\mathrm{III}}\right] \lambda_{1909}$ line, helium lines, and lines from $\mathrm{Ar}^{2+}, \mathrm{Ar}^{3+}$, and $\mathrm{Ne}^{2+}$. We fit with BEAGLE the integrated line fluxes, computed from the observed spectra as detailed in Section 2.2, with a model similar to the one described above, but letting the carbon-to-oxygen abundance ratio and depletion factor free to vary.
This model is therefore described by the seven free parameters $\boldsymbol{\Theta}^{\text {lines }}=\left[\log \left(M / \mathrm{M}_{\odot}\right), \log (t / \mathrm{yr}), \log \left(Z / \mathrm{Z}_{\odot}\right), \log U_{\mathrm{S}}, \hat{\tau}_{V}, \mathrm{C} / \mathrm{O}, \xi_{\mathrm{d}}\right]$.

The Bayesian approach adopted in BEAGLE allows us to derive the posterior probability distribution of the model parameters $\boldsymbol{\Theta}^{\text {phot+Balmer }}$ and $\boldsymbol{\Theta}^{\text {lines }}$,

$\mathrm{P}(\boldsymbol{\Theta} \mid \boldsymbol{D}, H) \propto \pi(\boldsymbol{\Theta}) \mathcal{L}(\boldsymbol{\Theta}),$,

where $\boldsymbol{D}$ indicates the data, $H$ the adopted model, $\pi(\boldsymbol{\Theta})$ the prior distribution, and $\mathcal{L}(\boldsymbol{\Theta})$ the likelihood function. We adopt independent priors for each parameter, uniform for $\log \left(M / \mathrm{M}_{\odot}\right)$, $\log \left(Z / \mathrm{Z}_{\odot}\right), \log U_{\mathrm{S}}, \xi_{\mathrm{d}}, \mathrm{C} / \mathrm{O}$, Gaussian for $\log (t / \mathrm{yr})$, and exponential for $\hat{\tau}_{V}$, and report the prior definitions and ranges in Table 2. We adopt a Gaussian likelihood function with independent errors 
Table 2. Free parameters of the model adopted in BEAGLE to fit the COS/SDSS photometry + Balmer emission lines [parameters $\log \left(M / \mathrm{M}_{\odot}\right), \log (t / \mathrm{yr})$, $\log \left(Z / Z_{\odot}\right), \log U_{\mathrm{S}}$, and $\left.\hat{\tau}_{V}\right]$, and to fit the emission line fluxes (all parameters in the table) of the 10 nearby star-forming galaxies analysed in this work. The symbol $\mathcal{U}$ indicates a uniform distribution in the specified range, $\mathcal{N}(\mu ; \sigma)$ a Gaussian (Normal) distribution with mean $\mu$ and standard deviation $\sigma$, truncated in the specified range.

\begin{tabular}{lcl}
\hline Parameter & \multicolumn{1}{c}{ Prior } & \multicolumn{1}{c}{ Description } \\
\hline $\log \left(M / \mathrm{M}_{\odot}\right)$ & $\mathcal{U} \in[1,9]$ & Stellar mass \\
$\log (t / \mathrm{yr})$ & $\mathcal{N}(7.5 ; 1.5) \in[7$, & Age of oldest stars in the \\
& $10.15]$ & \\
& $\mathcal{U} \in[-2.2,0.25]$ & $\begin{array}{l}\text { galaxy } \\
\text { Stellar and interstellar } \\
\text { metallicity }\end{array}$ \\
$\log \left(Z / \mathrm{Z}_{\odot}\right)$ & $\mathcal{U} \in[-4,-1]$ & $\begin{array}{l}\text { Effective gas ionization } \\
\text { parameter } \\
\log U_{\mathrm{S}}\end{array}$ \\
$\hat{\tau}_{V}$ & $\exp -\hat{\tau}_{V} \in[0,2]$ & $\begin{array}{l}V \text {-band attenuation } \\
\text { optical depth }\end{array}$ \\
$\mathrm{C} / \mathrm{O}$ & $\mathcal{U} \in[0.1,1]$ & $\begin{array}{l}\text { Carbon-to-oxygen ratio } \\
\text { Dust-to-metal mass ratio }\end{array}$ \\
$\xi_{\mathrm{d}}$ & $\mathcal{U} \in[0.1,0.5]$ & \\
\hline
\end{tabular}

on each measurement,

$$
-2 \ln \mathcal{L}(\boldsymbol{\Theta})=\sum_{i}\left[\frac{y_{i}-\hat{y}_{i}(\boldsymbol{\Theta})}{\sigma_{i}}\right]^{2},,
$$

where the summation index $i$ runs over all observables considered in the fitting, i.e. the Balmer lines and COS+SDSS photometry in one case, the emission lines listed in Table 1 in the other case. In equation (2), $y_{i}$ indicates the data, $\sigma_{i}$ the observational error, and $\hat{y}_{i}(\boldsymbol{\Theta})$ the model prediction. We adopt the Nested Sampling algorithm (Skilling et al. 2006) in the MULTINEST implementation (Feroz, Hobson \& Bridges 2009) to sample the posterior probability distribution of the free parameters of our model. We note that this implies that each model spectrum is computed in BEAGLE 'on-the-fly' for each combination of parameters selected by MULTINEST during the sampling of the posterior probability distribution of the model parameters.

\section{RESULTS}

The model we have adopted allows us to well reproduce all the observables here considered, i.e. the combination of COS/SDSs photometry and H-Balmer lines, and the emission lines of Table 1. In Table 1, we also report the emission line fluxes predicted by our model for each object fitted, while we show in Fig. 2 a visual example of the fitting of the object SB 80, an embedded H II region at $z=0.01085$. We show in Figs 1 (a) and (b), the line ratios predicted by BEAGLE for all fitted objects in our sample. Table 1 indicate a remarkable agreement between the predictions of our relatively simple model and the data.

\subsection{Physical properties of extreme star-forming regions}

Table 3 lists the physical properties of our sample derived from the BEAGLE-based emission lines fitting. As already shown in S17, our 10 galaxies cover a broad range of gas-phase metallicities $12+$ $\log (\mathrm{O} / \mathrm{H}) \sim 7.9-8.5\left[\log \left(\mathrm{Z} / \mathrm{Z}_{\odot}\right) \sim-0.8\right.$ to -0.2$]$ and show low dust attenuation values. We derive high ionization parameters in all galaxies [ $\left.\log U_{\mathrm{S}} \gtrsim-3\right]$, even in the highest metallicity ones, namely SB 179 and SB $191[12+\log (\mathrm{O} / \mathrm{H}) \sim 8.5]$, which show $\log U_{\mathrm{S}} \sim$ -2.60 and $\sim-2.14$, respectively. Galaxies showing intermediate metallicities and high ionization parameters are rare in the Local Universe (e.g. among sDss galaxies, see fig 2 of Carton et al. 2017), while they become more common at higher redshifts, as suggested by the detection of high-ionization metal lines at $z \gtrsim 5$ (e.g. Stark et al. 2015a; Mainali et al. 2017). The emission lines that we consider in this analysis originate from both refractory $(\mathrm{O}, \mathrm{C})$ and nonrefractory $(\mathrm{N}, \mathrm{S}, \mathrm{Ar}, \mathrm{Ne})$ elements, and this enables us to place tight constraints on the fraction of metals depleted onto dust grains $\xi_{d}$. The $\xi_{d}$ values reported in Table 3 are significantly lower than the Solar value $\xi_{\mathrm{d} \odot}=0.36$, indicating that most metals in these galaxies are in the gas phase and not locked into dust grains.

Fig. 3 compares the gas-phase metallicity $12+\log (\mathrm{O} / \mathrm{H})$ derived with our analysis with the values obtained by S17 using a standard method, the 'direct- $T_{\mathrm{e}}$ ' one (see their Section 3.2). S17 measure the electron temperature $\left(T_{\mathrm{e}}\right)$ of $\mathrm{O}^{+}$and $\mathrm{O}^{2+}$ using ratios of auroral ([O II] $\lambda \lambda 7320,7330$ and $[\mathrm{O} \mathrm{III}] \lambda 4363)$ and strong ([O II] $\lambda \lambda 3726,3729$ and [O III] $\lambda \lambda 4959,5007)$ lines, and then compute the abundances of the $\mathrm{O}^{+}$and $\mathrm{O}^{2+}$ ions using the strong lines. As Fig. 3 shows, the agreement between the $\mathrm{S} 17$, direct $-T_{\mathrm{e}}$ based estimates of $12+\log (\mathrm{O} / \mathrm{H})$, and our values (listed in Table 3) is generally good, although the BEAGLE-based metallicities are slightly larger than the $\mathrm{S} 17$ ones, by $\sim 0.05$ dex at $12+\log (\mathrm{O} / \mathrm{H}) \lesssim 8.3$, and by $\sim 0.2$ dex for the two galaxies with larger $12+\log (\mathrm{O} / \mathrm{H})$ values, SB 179 and SB 191. These two objects show large ionization parameters, and $\mathrm{S} 17$ ignore the contribution from $\mathrm{O}^{3+}$ when measuring the oxygen abundance, but they evaluate the contribution of $\mathrm{O}^{3+}$ to be minor by considering a relation between $\mathrm{O}^{3+}$ and $\mathrm{He}^{2+} / \mathrm{He}^{+}$(Izotov et al. 2006). The only object that does not follow the trend above is SB 198, for which our metallicity estimate is $\sim 0.3$ dex lower than the S17 one. The metallicity estimated for SB 198 , however, is the most uncertain, and the statistical significance of the difference among the two methods is $\sim 3 \sigma$, hence more data would be necessary to gain better insight into the origin of this discrepancy.

We note that differences between metallicities estimated with photoionization models and with the 'direct- $T_{\mathrm{e}}$ ' method, such as those discussed above, have been reported in several recent works (e.g. Blanc et al. 2015; Vale Asari et al. 2016; Pérez-Montero \& Amorín 2017). Interestingly, both Blanc et al. (2015) and Vale Asari et al. (2016) find that metallicities estimated with photoionization models are systematically larger (by $\sim 0.2-0.4$ dex) than those estimated with the direct- $T_{\mathrm{e}}$ method, qualitatively consistent with our findings, and that photoionization models can provide metallicity estimates in better agreement with those derived from metal recombination lines. In Vale Asari et al. (2016) (see their section 6.3) it is suggested that the [OIII] $\lambda 4363$ line is at the origin of the measured differences among photoionization- and direct- $T_{\mathrm{e}}$ based metallicities. The $[\mathrm{O}$ III $] \lambda 4363$ line is used to determine the electron temperature in the $\mathrm{O}^{2+}$ zone, but this line can be affected by temperature fluctuations and non-Maxwellian velocity distributions of the free electrons (Nicholls, Dopita \& Sutherland 2012). These effects can boost the $[\mathrm{O}$ III] $\lambda 4363$ line flux, thus modifying the derived electron temperature and, consequently, the metallicity. In this work, the differences among the photoionization- and direct$T_{\mathrm{e}}$-based metallicities are, for most objects, significantly smaller than found in previous works. The reason is likely related to the fact that $\mathrm{S} 17$ directly measure the $T_{\mathrm{e}}$ of both $\mathrm{O}^{+}$and $\mathrm{O}^{2+}$, instead of only measuring the temperature of $\mathrm{O}^{2+}$ and using calibrated relations to derive that of $\mathrm{O}^{+}$. The $\mathrm{S} 17$ approach can thus alleviate some of the limitations of the direct- $T_{\mathrm{e}}$ method, providing estimates in better agreement with those derived with the photoionization models incorporated into BEAGLE. 


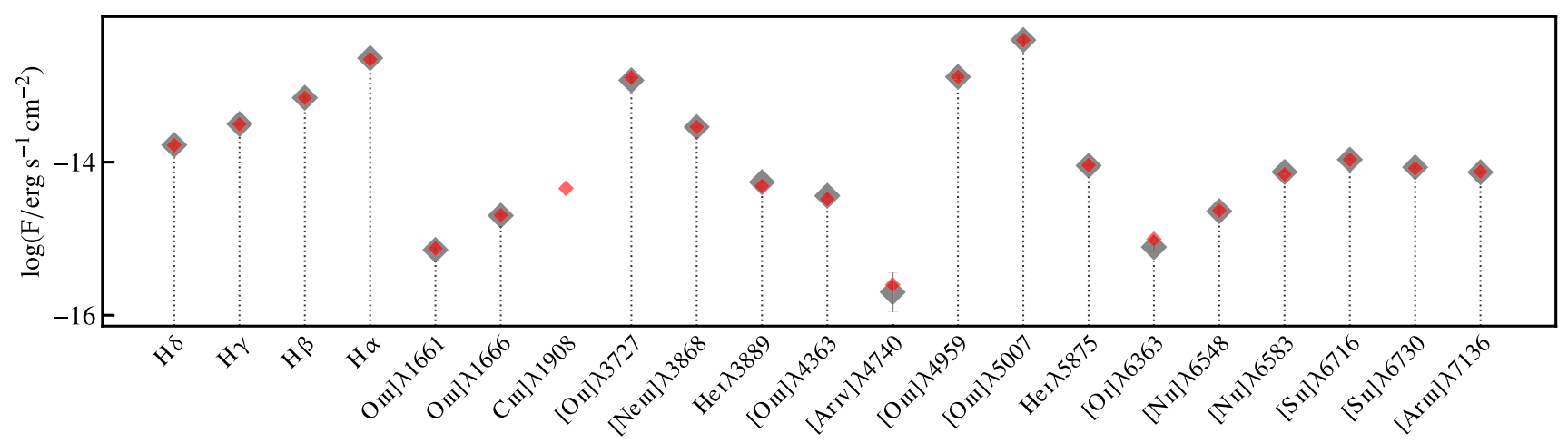

Figure 2. Example of a BEAGLE fitting of the galaxy SB 80, an embedded $\mathrm{H}_{\text {II }}$ region. Observed fluxes are indicated by grey diamonds, model fluxes by red ones. Note that in most cases the error bars on the observed fluxes are contained within the markers.

Table 3. Physical parameters of the 10 objects analysed in this work, obtained by fitting with BEAGLE the emission lines listed in Table 1 . We report the posterior median and 68 per cent central credible interval of stellar age $t$, gas-phase metallicity $12+\log (\mathrm{O} / \mathrm{H})$, ionization parameter $\log U_{\mathrm{S}}$, dust-to-metal mass ratio $\xi_{\mathrm{d}}$, and $V$-band attenuation optical depth $\hat{\tau}_{V}$.

\begin{tabular}{|c|c|c|c|c|c|}
\hline ID & $t / \mathrm{Myr}$ & $12+\log (\mathrm{O} / \mathrm{H})$ & $\log U_{\mathrm{S}}$ & $\xi_{\mathrm{d}}$ & $\hat{\tau}_{V}$ \\
\hline 36 & $1.11 \pm 0.1$ & $7.97 \pm 0.03$ & $-2.77 \pm 0.02$ & $0.19 \pm 0.04$ & $0.1 \pm 0.02$ \\
\hline 82 & $2.03 \pm 0.1$ & $7.95 \pm 0.01$ & $-2.36 \pm 0.02$ & $0.28 \pm 0.01$ & $0.18 \pm 0.008$ \\
\hline 110 & $11.06 \pm 4$ & $8.15 \pm 0.03$ & $-2.66 \pm 0.02$ & $0.35 \pm 0.03$ & $0.04 \pm 0.02$ \\
\hline 111 & $1.27 \pm 0.3$ & $7.90 \pm 0.03$ & $-2.68 \pm 0.02$ & $0.18 \pm 0.04$ & $0.04 \pm 0.02$ \\
\hline 191 & $3.15 \pm 0.2$ & $8.47 \pm 0.01$ & $-2.14 \pm 0.03$ & $0.12 \pm 0.02$ & $0.00 \pm 0.005$ \\
\hline 198 & $7.05 \pm 2$ & $8.20 \pm 0.08$ & $-2.95 \pm 0.06$ & $0.12 \pm 0.02$ & $0.02 \pm 0.02$ \\
\hline
\end{tabular}

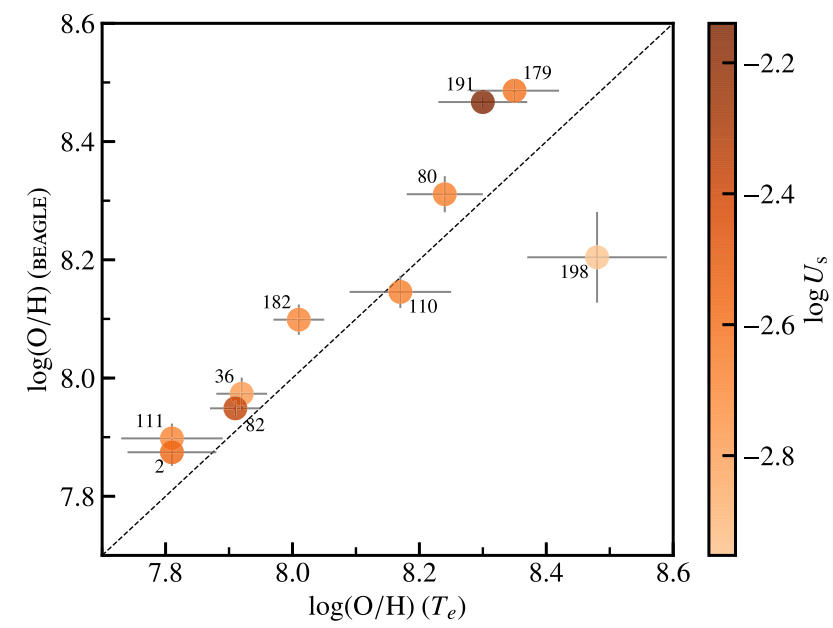

Figure 3. Comparison between the gas-phase metallicity $12+\log (\mathrm{O} / \mathrm{H})$ obtained in this work with the BEAGLE tool ( $y$-axis) and by S17 with the 'direct- $T_{\mathrm{e}}$ ' method. Colour coded is the ionization parameter $\log U_{\mathrm{S}}$ obtained with the BEAGLE analysis. Error bars indicate the 68 per cent central credible interval.

\subsection{Production rate of $\mathbf{H}$-ionizing photons}

In this paper, we define $\xi_{\text {ion }}^{\star}=\dot{N}_{\text {ion }} / L_{\mathrm{UV}}^{\star}$ as the ratio between the production rate of $H$-ionizing photons $\left(\dot{N}_{\mathrm{ion}}\right)$ and the intrinsic stellar monochromatic $U$ V luminosity $\left(L_{\mathrm{UV}}^{\star}\right)$. The quantity $L_{\mathrm{UV}}^{\star}$ is computed from the pure stellar spectrum considering a flat filter centred at $1500 \AA$ and of $100 \AA$ width (e.g. see Robertson et al. 2013). $L_{\mathrm{UV}}^{\star}$ is the emission from stars that would be observed in the absence of gas and dust in galaxies, i.e. neither it includes the effect of absorption and emission from neutral and photoionized gas, nor of dust attenuation. Previous studies have adopted different definitions of the monochromatic UV luminosity, for example, by considering the stellar + nebular luminosity $L_{\mathrm{UV}}^{\mathrm{H} \text { II }}$, corrected for dust attenuation outside $\mathrm{H}_{\text {II }}$ regions but not for dust attenuation inside $\mathrm{H}_{\text {II }}$ regions nor for recombination-continuum emission, or the observed luminosity $L_{\mathrm{UV}}$. For the sake of clarity, we introduce here the symbols $\xi_{\text {ion }}^{\mathrm{H} \text { II }}$ and $\xi_{\text {ion }}$ to differentiate between our definition of $\xi_{\text {ion }}^{\star}$ and the ratios $\xi_{\text {ion }}^{\mathrm{HI}}=\dot{N}_{\text {ion }} / L_{\mathrm{UV}}^{\mathrm{HII}}$ and $\xi_{\text {ion }}=\dot{N}_{\text {ion }} / L_{\mathrm{UV}}$.

To compute $\xi_{\text {ion }}^{\star}$, we combine the monochromatic UV luminosity $\left(L_{\mathrm{UV}}^{\star}\right)$ inferred from the fitting to COS/SDSS photometry + Balmer lines with the rate of H-ionizing photons $\left(\dot{N}_{\text {ion }}\right)$ obtained by fitting the different UV and optical emission lines. We report in Table 4 the $\xi_{\text {ion }}^{\star}$ values for the 10 galaxies in our sample. Our galaxies exhibit $\log \left(\xi_{\text {ion }}^{\star} / \mathrm{erg}^{-1} \mathrm{~Hz}\right)$ values in the range from $\sim 25.2$ to $\sim 25.8$, i.e. generally larger then the values adopted in standard reionization models (25.2-25.4). We also report in Table 4 the values we derive for $\xi_{\text {ion }}^{\mathrm{HII}}$ and $\xi_{\text {ion }}$, to highlight the impact that different definitions of this quantity can have on the quoted values. In particular, $\xi_{\text {ion }}^{\mathrm{H} \text { II }}$ can be either smaller or larger than $\xi_{\text {ion }}^{\star}$, depending on the relative strengths of dust attenuation inside $\mathrm{H}_{\text {II }}$ regions (which lowers $L_{\mathrm{UV}}^{\mathrm{HII}}$ and raises $\xi_{\text {ion }}^{\mathrm{HI}}$; see e.g. object SB 191) and recombination-continuum emission (which boosts $L_{\mathrm{UV}}^{\mathrm{HII}}$ and hence lowers $\xi_{\text {ion }}^{\mathrm{H}}$; see e.g. SB 2). Considering the observed (i.e. attenuated) UV luminosity $L_{\mathrm{UV}}$ generally implies larger $\xi_{\text {ion }}$ values, but the difference between $\xi_{\text {ion }}^{\star}$ and $\xi_{\text {ion }}$ depends on the amount of 
Table 4. Production rate of H-ionizing photons per unit monochromatic UV luminosity [expressed in units of $\log \left(\mathrm{erg}^{-1} \mathrm{~Hz}\right)$ ]. We report different definitions of this quantity, using the intrinsic stellar luminosity $\left(\xi_{\text {ion }}^{\star}\right)$, the stellar + nebular luminosity $\left(\xi_{\text {ion }}^{\mathrm{HI}}\right)$, and the observed (i.e. attenuated) luminosity ( $\xi_{\text {ion }}$; see Section 3.2). The reported values correspond to the posterior median, while the errors indicate to the 68 per cent central credible interval.

\begin{tabular}{lccc}
\hline ID & $\log \xi_{\text {ion }}^{*}$ & $\log \xi_{\text {ion }}^{\mathrm{HI}}$ & $\log \xi_{\text {ion }}$ \\
\hline 2 & $25.84 \pm 0.05$ & $25.81 \pm 0.11$ & $26.42 \pm 0.10$ \\
36 & $25.37 \pm 0.02$ & $25.36 \pm 0.03$ & $25.38 \pm 0.04$ \\
80 & $25.73 \pm 0.01$ & $25.69 \pm 0.01$ & $26.26 \pm 0.03$ \\
82 & $25.80 \pm 0.01$ & $25.84 \pm 0.02$ & $25.85 \pm 0.02$ \\
110 & $25.24 \pm 0.02$ & $25.26 \pm 0.03$ & $25.27 \pm 0.04$ \\
111 & $25.38 \pm 0.03$ & $25.37 \pm 0.03$ & $25.47 \pm 0.04$ \\
179 & $25.50 \pm 0.03$ & $25.52 \pm 0.04$ & $25.84 \pm 0.03$ \\
182 & $25.57 \pm 0.02$ & $25.61 \pm 0.03$ & $25.73 \pm 0.05$ \\
191 & $25.82 \pm 0.02$ & $25.94 \pm 0.04$ & $26.06 \pm 0.03$ \\
198 & $25.65 \pm 0.02$ & $25.60 \pm 0.03$ & $25.63 \pm 0.03$ \\
\hline
\end{tabular}

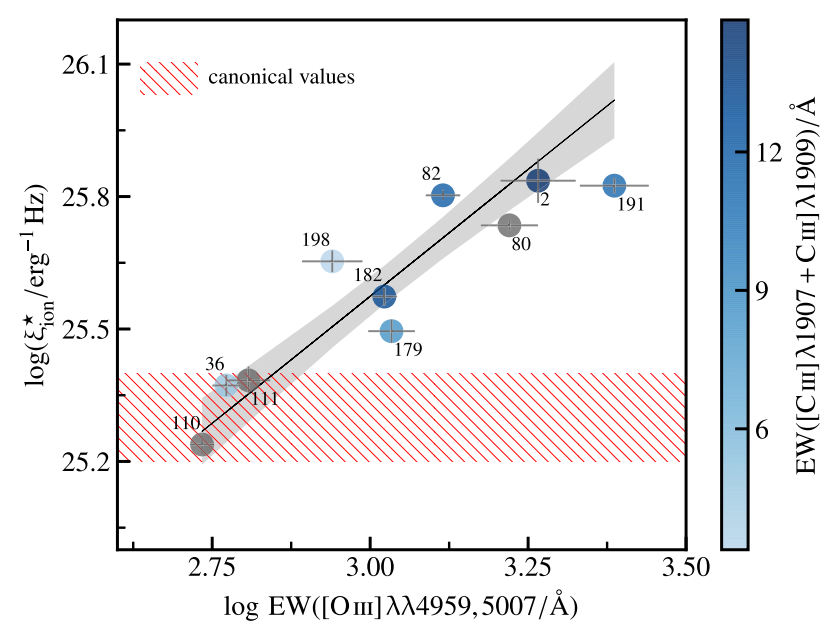

Figure 4. Relation between production rate of $\mathrm{H}$-ionizing photons per unit intrinsic monochromatic UV luminosity ( $y$-axis) and equivalent width of the [OIII] $\lambda \lambda 4959,5007$ doublet ( $x$-axis). Colour-coded is the equivalent width of [CIII] $\left.\lambda 1907+\mathrm{C}_{\mathrm{III}}\right] \lambda 1909$, while grey circles indicate objects with no $\left.\left[\mathrm{C}_{\text {III }}\right] \lambda 1907+\mathrm{C}_{\text {III }}\right] \lambda 1909$ detections. Error-bars on the circles indicate the 68 per cent central credible region. The black line indicates the best-fitting linear relation between $\xi_{\text {ion }}^{\star}$ and $\mathrm{EW}\left(\left[\mathrm{O}_{\mathrm{III}}\right]\right.$ ) reported in equation (3), while the grey bands indicate the 68 per cent credible region. The red hatched region indicates typical $\xi_{\text {ion }}^{\star}$ values adopted in reionization models.

dust attenuation affecting stellar and nebular emission outside $\mathrm{H}$ II regions.

In Fig. 4, we show the relation between $\xi_{\text {ion }}^{\star}$ and equivalent width of the [O III] $\lambda \lambda 44959,5007$ doublet. Objects exhibiting larger $\mathrm{EW}([\mathrm{O} \mathrm{III}])$ also show larger $\xi_{\text {ion }}^{\star}$. A relation between the two quantities is expected, since large [O III] $\lambda \lambda 4959,5007$ equivalent widths are typically produced by stellar populations with young ages and sub-solar metallicities, which would also produce copious amounts of H-ionizing photons. Moreover, part of the small scatter visible in Fig. 4 can be ascribed to the relatively narrow range of [O III $] \lambda \lambda 4959,5007 / \mathrm{H}, \beta$ probed by our objects (Fig. 1a). Remarkably, the tight relation in Fig. 4 also implies a limited variation of ionized-gas properties in our objects, since the relation between $\xi_{\text {ion }}^{\star}$ and $\mathrm{EW}\left(\left[\mathrm{O}_{\mathrm{III}}\right]\right)$ depends on the shape of the ionizing spectrum (e.g. stellar age and metallicity), ionization parameter (geometry of the photoionized regions), metal abundances, and gas density. The colour coding of the circles in Fig. 4 further indicates the equivalent width of $[\mathrm{C}$ III $] \lambda 1907+\mathrm{C}$ III $] \lambda 1909$ measured from the COS spectra. As noted by S17 (their fig. 10), the equivalent widths of [C III] $\left.\lambda 1907+\mathrm{C}_{\mathrm{III}}\right] \lambda 1909$ and $[\mathrm{O} \mathrm{III}] \lambda \lambda 4959,5007$ are related to each other, the most extreme $\left.\mathrm{C}_{\mathrm{III}}\right]$ emitters showing largest [O $\left.\mathrm{III}\right]$ emission. Additional high-quality rest-frame UV spectra are required to calibrate a relation between $\xi_{\text {ion }}^{\star}$ and $\mathrm{C}$ III], but this relation has the potential to allow indirect constraints on $\xi_{\text {ion }}^{\star}$ even for galaxies deep into the EoR.

We fit the relation between $\xi_{\text {ion }}^{\star}$ and EW([O III]) using 'Orthogonal Distance Regression', a linear regression method which allows us to account for errors along both the $y$ - and $x$-axis. ${ }^{7}$ The black line in Fig. 4 show the best-fitting relation

$$
\begin{aligned}
\log \left(\xi_{\text {ion }}^{\star} / \mathrm{erg}^{-1} \mathrm{~Hz}\right)= & 22.12 \pm 0.48+1.15 \pm 0.16 \\
& \times \log (\mathrm{EW}[\mathrm{O} \text { III }] / \AA),,
\end{aligned}
$$

while the grey bands indicate the 68 per cent credible region around the best-fitting relation. We also fit a relation considering the combined equivalent widths of $\mathrm{H}, \beta+[\mathrm{O}$ III $] \lambda \lambda 4959,5007$, obtaining a relation with intercept $($ slope $)=21.95$ (1.18) instead of 22.12 (1.15). Such a relation is particularly useful to convert in a physicallymotivated way broad-band colour excesses (e.g. IRAC excess; Shim et al. 2011; Stark et al. 2013; Smit et al. 2014) into $\xi_{\text {ion }}^{\star}$ values.

\section{DISCUSSION}

The results presented in the previous section allow us to draw several conclusions regarding our modelling approach. The BEAGLE-based fitting of the UV and optical emission lines, and in particular our ability to match simultaneously the emission from different ionization states of the same element (e.g. $\mathrm{O}^{+}$and $\mathrm{O}^{2+} ; \mathrm{Ar}^{2+}$ and $\mathrm{Ar}^{3+}$ ), ions with widely different ionizing energies (e.g. $27.6 \mathrm{eV}$ for $\mathrm{Ar}^{2+}$ and $41.0 \mathrm{eV}$ for $\mathrm{Ne}^{3+}$ ) and electron temperature-sensitive ratios (e.g. [O III] $\lambda 4363 /[\mathrm{O}$ III] $\lambda \lambda 4959,5007)$, suggests that the ionizing stellar-population prescription and nebular parametrization of our model are suited to interpret observations of extreme star-forming regions. The matching of the different $\mathrm{H}$-Balmer lines validates our adoption of an SMC extinction curve to model dust attenuation, and that of the $\left[\mathrm{S}_{\mathrm{II}}\right] \lambda \lambda 6716,6731$ line doublet the gas density we have adopted. Also, the ability of our model to match emission lines of different elements $(\mathrm{C}, \mathrm{N}, \mathrm{O}, \mathrm{Ne}, \mathrm{S}$, and $\mathrm{Ar}$ ) suggests that these galaxies show similar metal abundance ratios, which we can reproduce by varying only the interstellar metallicity, dust depletion factor, and carbon-to-oxygen ratio.

We note, however, that in our analysis we did not attempt to fit the He II recombination lines (He II $\lambda 1640$ and $\mathrm{He}$ II $\lambda 4686$ ). As we have discussed in S17, the stellar population + photoionization models incorporated in BEAGLE are unable to reproduce the very large equivalent widths of the He II $\lambda 1640$ nebular line (up to $\sim 1.7 \AA$ ) observed in the galaxies of our sample that do not show WR spectral features, i.e. galaxies with metallicities $12+\log (\mathrm{O} / \mathrm{H}) \lesssim 8$. As discussed in Shirazi \& Brinchmann 2012, this problem is common to other widely used stellar population models, which fail in providing enough $\mathrm{He}^{+}$ionizing photons for stellar populations with $Z / \mathrm{Z}_{\odot} \lesssim 0.2$. Several potential sources of additional $\mathrm{He}^{+}$ionizing photons have been proposed in the literature, such as X-ray binaries, fast radiative shocks, and low-luminosity AGNs (e.g. Stasińska et al. 2015). As discussed in section 5.3 of S17, data on our galaxies do not provide evidence for the presence of X-ray binaries or fast radiative shocks, although deeper X-ray observations and larger

\footnotetext{
${ }^{7}$ https://docs.scipy.org/doc/scipy/reference/odr.html
} 
grids of shock models (extending to lower metallicities) would be required to rule out such presence. Thanks to the recent incorporation into BEAGLE of the AGN models for Narrow Line Region emission of Feltre, Charlot \& Gutkin (2016), in future work we will test the possibility of low-luminosity AGNs to explain the observed HeII $\lambda 1640$ nebular emission of our non-WR galaxies.

The constraints obtained with our BEAGLE-based modelling of emission lines + photometry on $\xi_{\text {ion }}^{\star}$ have potentially important implications for our understanding of the sources of $\mathrm{H}$ ionizing photons in pristine star-forming galaxies. We find that $\xi_{\text {ion }}^{\star}$ can reach values significantly larger than those considered in many reionization models, especially for galaxies exhibiting $\mathrm{EW}([\mathrm{O} \mathrm{III}] \lambda \lambda 4959,5007) \gtrsim 1000$. Such galaxies are relatively rare at low redshifts, while they become much more common at higher ones. Very large equivalent widths of optical emission lines are routinely inferred from contamination of broad-band Spitzer/IRAC photometry, corresponding to $\mathrm{EW}(\mathrm{H}, \alpha+[\mathrm{N}$ II $] \lambda \lambda 6548,6584+$ [S II] $\lambda \lambda 6716,6731$ ) up to $\sim 400$ for galaxies at $z \sim 4.5$ (Stark et al. 2013; Mármol-Queraltó et al. 2016; Smit et al. 2016), reaching $\gtrsim 1000$ for galaxies at $z \sim 5.2$ (Rasappu et al. 2016). Smit et al. (2014) derive $\mathrm{EW}\left(\mathrm{H}, \beta+\left[\mathrm{O}_{\mathrm{III}}\right] \lambda \lambda 4959,5007\right) \gtrsim 1000$ for extremely blue IRAC galaxies at $z \sim 7$. Observations of highionization UV lines provide a consistent picture: while high equivalent widths of UV lines, such as $\mathrm{C}_{\mathrm{IV}} \lambda \lambda 1548,1551$, [C I]tex304 $\lambda 1907+$ CIII] $\lambda 1909$, and OIII] $\lambda \lambda 1660,1666$, are rare at low redshift, they have been routinely detected at higher redshifts (e.g. Stark et al. 2015a,b, 2017; Mainali et al. 2017). The emerging picture is that at $z \gtrsim 6$, a combination of high star formation rates per unit stellar mass, young stellar populations, low metallicities, and high ionization parameters favours the presence of $\mathrm{EW} \gtrsim 1000$ (10) for the brightest optical (UV) lines, which our analysis suggests correspond to values of $\xi_{\text {ion }}^{\star}$ significantly larger than canonical values used in reionization models. From a theoretical perspective, simulations indicate that the escape fraction of $\mathrm{H}$-ionizing photons from galaxies is timedependent, being tightly coupled to the energy input from stellar feedback (winds, $\mathrm{SNe}$ ), which opens 'clear' channels through which ionizing radiation can escape (e.g. Wise \& Cen 2009; Trebitsch et al. 2017). The presence of broad components in the emission lines of our objects suggests that such feedback mechanisms are at work in star-forming regions with a high production efficiency of $\mathrm{H}$-ionizing photons. If regions with high $\xi_{\text {ion }}^{\star}$ also exhibit large $f_{\text {esc }}$, then short, powerful bursts of star formation in low-metallicity, lowmass galaxies can provide enough $\mathrm{H}$-ionizing photons to achieve the time-averaged value $\log \left(f_{\text {esc }} \xi_{\text {ion }}^{\star} / \mathrm{erg}^{-1} \mathrm{~Hz}\right)=24.5$ (Bouwens et al. 2016) necessary to keep the IGM ionized. ${ }^{8}$

\section{COMPARISON WITH PREVIOUS WORK}

The importance of constraining the production rate of $\mathrm{H}$-ionizing photons to understand the role of star-forming galaxies in cosmic reionization motivated several recent studies in which this quantity has been estimated using different observables. Bouwens et al. (2016) consider star-forming galaxies at $z \sim 3.8-5.4$ in the GOODS North and South fields, and estimate the production rate of $\mathrm{H}$-ionizing photons $\dot{N}_{\text {ion }}$ from the contamination of $\mathrm{H}, \alpha+\left[\mathrm{N}_{\mathrm{II}}\right] \lambda \lambda 6548,6584+\left[\mathrm{S}_{\mathrm{II}}\right] \lambda \lambda 6716,6731$ to broad-band photometry. In practice, they derive the $\mathrm{H} \alpha$ luminosity by adopting fixed ratios of $[\mathrm{N} I I] / \mathrm{H}, \alpha$ and $\left[\mathrm{S}_{\mathrm{II}}\right] / \mathrm{H}, \alpha$, and estimate the

${ }^{8}$ This value assumes a minimum UV magnitude $M_{1500}=-13$, while adopting $M_{1500}=-17$ would imply a higher $\log \left(f_{\text {esc }} \xi_{\text {ion }}^{\star} / \mathrm{erg}^{-1} \mathrm{~Hz}\right)=24.9$. amount of dust attenuation from the UV-continuum slope. They then derive a dust-corrected UV luminosity $L_{\mathrm{UV}}^{\mathrm{HII}}$. The $\xi_{\text {ion }}^{\mathrm{H} \text { II }}$ they obtain spans a broad range of values $\log \left(\xi_{\text {ion }}^{\mathrm{HII}} / \mathrm{erg}^{-1} \mathrm{~Hz}\right) \sim 24.2-26$, compatible with the values spanned by our galaxies, with the bulk of their galaxies showing values $\log \left(\xi_{\text {ion }}^{\mathrm{H} \text { II }} / \mathrm{erg}^{-1} \mathrm{~Hz}\right) \sim 25-25.6$, and the bluest and less luminous galaxies exhibiting the highest values.

Schaerer et al. (2016) analyse five LyC-leaking galaxies at $z \sim$ 0.3 with large $\operatorname{EW}\left(\left[\mathrm{O}_{\mathrm{III}}\right]\right) \gtrsim 1000$, deriving $\dot{N}_{\text {ion }}$ from the (dustcorrected) $\mathrm{H} \beta$ luminosity, and $L_{\mathrm{UV}}^{\mathrm{HII}}$ from fitting the UV (GALEX) and optical (SDSS) photometry of the galaxies. As in Bouwens et al. (2016) and Schaerer et al. (2016) derive a dust-corrected UV luminosity, but without correcting for the contribution from recombination continuum and dust within $\mathrm{H}$ II regions. The values they obtain are in the range $\log \left(\xi_{\text {ion }}^{\mathrm{H}} / \mathrm{erg}^{-1} \mathrm{~Hz}\right) \sim 25.1-25.5$, generally lower than the values we derive for galaxies with comparable EW([O III]).

Matthee et al. (2017) study a sample of $\sim 600 \mathrm{H} \alpha$ emitters at $z=$ 2.2 from the HiZELS survey (Geach et al. 2008). They measure the $\mathrm{H} \alpha$ luminosity from a narrow photometric band centred on $\mathrm{H}, \alpha+$ $\left[\mathrm{N}_{\text {II }}\right] \lambda \lambda 6548,6584$, correcting $\mathrm{H} \alpha$ for the $\left[\mathrm{N}_{\text {II] }}\right] \lambda \lambda 6548,6584$ contribution. They derive dust corrections with different methods, and compute $\dot{N}_{\text {ion }}$ from the dust-corrected $\mathrm{H} \alpha$ luminosity. The $\xi_{\text {ion }}^{\mathrm{HI}}$ they derive covers a broad range of values $\log \left(\xi_{\text {ion }}^{\mathrm{HI}} / \mathrm{erg}^{-1} \mathrm{~Hz}\right) \sim 24-26$, with the exact values being sensitive to the adopted dust correction method. They find a strong correlation among $\xi_{\text {ion }}^{\mathrm{HII}}$ and $\mathrm{EW}(\mathrm{H}, \alpha)$, with the highest equivalent width galaxies exhibiting the large $\xi_{\text {ion }}^{\mathrm{HII}}$ values.

Izotov et al. (2017a) study a sample of $\sim 14000$ compact starforming galaxies at $z \leq 1$ from SDSS DR12. They derive dust corrections from Balmer line ratios, compute $\dot{N}_{\text {ion }}$ from the dustcorrected $\mathrm{H} \beta$ luminosity, and obtain the monochromatic UV luminosity from SED fitting to GALEX and SDSS photometry. They derive $\xi_{\text {ion }}^{\mathrm{H} \text { II }}$ values in the range $\log \left(\xi_{\text {ion }}^{\mathrm{H} \text { II }} / \mathrm{erg}^{-1} \mathrm{~Hz}\right) \sim 24.4-25.6$, with the bluest galaxies exhibiting the largest $\xi_{\text {ion }}^{\mathrm{H} \text { II }}$ values.

Shivaei et al. (2018) consider a large sample of $\sim 700$ galaxies at redshift $z \sim 1.4-2.6$ from the MOSDEF survey (Kriek et al. 2015). They derive $\dot{N}_{\text {ion }}$ from the dust-corrected $\mathrm{H} \alpha$ luminosity, and obtain $L_{\mathrm{UV}}^{\mathrm{HII}}$ by fitting the 3D-HST multi-band photometry with the Bruzual \& Charlot (2003) population synthesis code. They apply average corrections to $\dot{N}_{\text {ion }}$ to account for a non-zero escape fraction of $\mathrm{H}$-ionizing photons, and find $\log \left(\xi_{\text {ion }}^{\mathrm{HI}} / \mathrm{erg}^{-1} \mathrm{~Hz}\right)$ values in the range from $\sim 25.00$ to $\sim 25.6$ (assuming an SMC extinction curve). As found by Bouwens et al. (2016) and Izotov et al. (2017a), they also find that the bluest galaxies exhibit the largest $\xi_{\text {ion }}^{\text {HI }}$ values. Measurements of the production rate of $\mathrm{H}$-ionizing photons in EoR galaxies are difficult because of the challenges in performing spectroscopic observations of such galaxies with existing facilities. Stark et al. $(2015 b, 2017)$ present detections of Ly $\alpha$, $\mathrm{C}_{\text {IV }} \lambda \lambda 1548,1551$ and $\left.\left[\mathrm{C}_{\mathrm{III}}\right] \lambda 1907+\mathrm{C}_{\mathrm{III}}\right] \lambda 1909$ in three galaxies at $z \gtrsim 7$, and estimate $\xi_{\text {ion }}^{\star}$ from the stellar populations + photoionization modelling of their UV emission lines and photometry. They derive $\log \left(\xi_{\text {ion }}^{\star} / \mathrm{erg}^{-1} \mathrm{~Hz}\right) \sim 25.6-25.8$, comparable to the largest values obtained in this work.

\section{CONCLUSIONS}

We have analysed a sample of 10 extreme nearby star-forming regions with high-quality UV-to-optical spectra from $H S T / C O S$, MMT, and SDSs. Our objects are characterized by low metallicities and high ionization parameters, as suggested by the locus they occupy in the [NII]-BPT diagram and in the O32 versus R23 one. Using a combination of stellar population + photoionization 
models incorporated into the BEAGLE spectral analysis tool, we have fitted a combination of UV-to-optical photometry + Balmer lines, and a broad set of UV and optical emission lines sensitive to the ionizing spectrum, ionization parameter, electrons temperature and density, metal abundances, and dust attenuation. This modelling has allowed us to constrain the intrinsic UV luminosity of the galaxies $L_{\mathrm{UV}}^{\star}$, their production rate of $\mathrm{H}$-ionizing photons, and their physical properties, such as gas-phase and stellar metallicities, ionization parameters, dust depletion factors, stellar ages, and dust attenuation.

The 10 galaxies of this work are characterized by large ionization parameters $\left(\log U_{\mathrm{S}} \gtrsim-3\right)$, low dust depletion factors $\left(\xi_{\mathrm{d}} \sim\right.$ $0.1-0.3)$, low dust attenuation and very young stellar ages $(t$ $\lesssim 10 \mathrm{Myr}$ in all but one case). As already shown in S17, our galaxies span a broad range of gas-phase metallicities $12+$ $\log (\mathrm{O} / \mathrm{H}) \sim 7.9-8.5$. We have compared the $12+\log (\mathrm{O} / \mathrm{H})$ values estimated with BEAGLE with those estimated in S17 with the direct- $T_{\mathrm{e}}$ method, finding an overall good agreement among the estimates, although the BEAGLE-based metallicities are systematically larger (by $\sim 0.05 \mathrm{dex}$ ) than the $\mathrm{S} 17$ ones. Larger differences $(\sim 0.2$ dex $)$ among the two methods appear at $12+\log (\mathrm{O} / \mathrm{H}) \gtrsim 8.3$. This confirms previous findings that metallicities derived with photoionization models are systematically larger than those derived with the direct- $T_{\mathrm{e}}$ method, although the differences found in this work are significantly smaller than those reported in the literature.

The production rate of $\mathrm{H}$-ionizing photons per unit (intrinsic) UV luminosity of our galaxies spans a wide range $\left[\log \left(\xi_{\text {ion }}^{\star} / \mathrm{erg}^{-1} \mathrm{~Hz}\right) \sim\right.$ $25.2-25.8$ ] compatible with the values measured in previous works, but with a strong dependence of $\xi_{\text {ion }}^{\star}$ on the equivalent width of the $[\mathrm{O}$ III $] \lambda \lambda 4959,5007$ line, such that high-EW([O III]) galaxies exhibit larger $\xi_{\text {ion }}^{\star}$ values. Such a relation is not completely surprising, since high $\mathrm{EW}([\mathrm{O} \mathrm{III}])$ are typically associated with young, metalpoor stellar populations, which are expected to emit large amounts of H-ionizing photons. Energy injection from young stellar populations (i.e. stellar 'feedback') is also expected to create clear channels in the ISM through which ionizing radiation can escape, and it is likely the cause of the low-intensity broad components emission lines observed in our galaxies. The combination of large $\xi_{\text {ion }}^{\star}$ and $f_{\text {esc }}$ in short bursts of star formation in low-mass galaxies can hence supply large quantities of $\mathrm{H}$-ionizing photons to the IGM. To enable more accurate determinations of $\xi_{\text {ion }}^{\star}$ in EoR galaxies, we have calibrated a new relation between $\xi_{\text {ion }}^{\star}$ and EW [O III] $\lambda \lambda 4959,5007$ $(\mathrm{EW}[\mathrm{O}$ III $] \lambda \lambda 4959,5007+\mathrm{EW} \mathrm{H}, \beta)$. This relation can be used to derive $\xi_{\text {ion }}^{\star}$ from broad-band observations of galaxies at $z \gtrsim 6$, e.g. from existing Spitzer/IRAC observations and future JWST/NIRCam ones, and from spectroscopic observations of $\left[\mathrm{O}_{\mathrm{III}}\right] \lambda \lambda 4959,5007$ with $J W S T /$ NIRSpec out to redshift $\sim 9.5$.

\section{ACKNOWLEDGEMENTS}

The authors are grateful to the referee, G. Stasińska, for several comments that significantly improved the paper. JC, SC, and AVG acknowledge support from the European Research Council (ERC) via an Advanced Grant under grant agreement no. 321323-NEOGAL. DPS acknowledges support from the National Science Foundation through the grant AST-1410155. TJ acknowledges support from NASA through Hubble Fellowship grant HST-HF2-51359.001-A awarded by the Space Telescope Science Institute. AF acknowledges support from the ERC via an Advanced Grant under grant agreement no. 339659-MUSICOS. The data described in this pa- per were obtained by the MMT Observatory, a joint facility of the University of Arizona and the Smithsonian Institution, and the NASA/ESA Hubble Space Telescope. Support for HST GO program \#14168 was provided by NASA through a grant from the Space Telescope Science Institute, which is operated by the Association of Universities for Research in Astronomy, Inc., diunder NASA contract NAS 5-26555.

\section{REFERENCES}

Abazajian K. N. et al., 2009, ApJS, 182, 543

Amorín R., Vílchez J. M., Hägele G. F., Firpo V., Pérez-Montero E., Papaderos P., 2012, ApJ, 754, L22

Atek H. et al., 2015a, ApJ, 800, 18

Atek H. et al., 2015b, ApJ, 814, 69

Baldwin J. A., Phillips M. M., Terlevich R., 1981, PASP, 93, 5

Blanc G. A., Kewley L., Vogt F. P. A., Dopita M. A., 2015, ApJ, 798, 99

Bouwens R. J. et al., 2015a, ApJ, 803, 34

Bouwens R. J., Illingworth G. D., Oesch P. A., Caruana J., Holwerda B., Smit R., Wilkins S., 2015b, ApJ, 811, 140

Bouwens R. J., Smit R., Labbé I., Franx M., Caruana J., Oesch P., Stefanon M., Rasappu N., 2016, ApJ, 831, 176

Bromm V., Yoshida N., 2011, ARA\&A, 49, 373

Bruzual G., Charlot S., 2003, MNRAS, 344, 1000

Carton D. et al., 2017, MNRAS, 468, 2140

Chabrier G., 2003, PASP, 115, 763

Chevallard J., Charlot S., 2016, MNRAS, 462, 1415

Fan X. et al., 2006, AJ, 132, 117

Feltre A., Charlot S., Gutkin J., 2016, MNRAS, 456, 3354

Ferland G. J. et al., 2013, Rev. Mex. Astron. Astrofis., 49, 137

Feroz F., Hobson M. P., Bridges M., 2009, MNRAS, 398, 1601

Finkelstein S. L. et al., 2015, ApJ, 810, 71

Fitzpatrick E. L., 1999, PASP, 111, 63

Geach J. E., Smail I., Best P. N., Kurk J., Casali M., Ivison R. J., Coppin K., 2008, MNRAS, 388, 1473

Gutkin J., Charlot S., Bruzual G., 2016, MNRAS, 462, 1757

Hirschmann M., Charlot S., Feltre A., Naab T., Choi E., Ostriker J. P., Somerville R. S., 2017, MNRAS, 472, 2468

Ishigaki M., Kawamata R., Ouchi M., Oguri M., Shimasaku K., Ono Y., 2018, ApJ, 854, 73

Izotov Y. I., Thuan T. X., 2008, ApJ, 687, 133

Izotov Y. I., Stasińska G., Meynet G., Guseva N. G., Thuan T. X., 2006, A\&A, 448, 955

Izotov Y. I., Thuan T. X., Guseva N. G., 2007, ApJ, 671, 1297

Izotov Y. I., Guseva N. G., Fricke K. J., Stasińska G., Henkel C., Papaderos P., 2010, A\&A, 517, A90

Izotov Y. I., Schaerer D., Thuan T. X., Worseck G., Guseva N. G., Orlitová I., Verhamme A., 2016, MNRAS, 461, 3683

Izotov Y. I., Guseva N. G., Fricke K. J., Henkel C., Schaerer D., 2017a, MNRAS, 467, 4118

Izotov Y. I., Thuan T. X., Guseva N. G., 2017b, MNRAS, 471, 548

James B. L., Tsamis Y. G., Barlow M. J., Westmoquette M. S., Walsh J. R., Cuisinier F., Exter K. M., 2009, MNRAS, 398, 2

Jaskot A. E., Oey M. S., 2013, ApJ, 766, 91

Kriek M. et al., 2015, ApJS, 218, 15

Kuhlen M., Faucher-Giguère C.-A., 2012, MNRAS, 423, 862

Mainali R., Kollmeier J. A., Stark D. P., Simcoe R. A., Walth G., Newman A. B., Miller D. R., 2017, ApJ, 836, L14

Mármol-Queraltó E., McLure R. J., Cullen F., Dunlop J. S., Fontana A., McLeod D. J., 2016, MNRAS, 460, 3587

Matthee J., Sobral D., Best P., Khostovan A. A., Oteo I., Bouwens R., Röttgering H., 2017, MNRAS, 465, 3637

Mirabel I. F., Dijkstra M., Laurent P., Loeb A., Pritchard J. R., 2011, A\&A, 528, A149

Nicholls D. C., Dopita M. A., Sutherland R. S., 2012, ApJ, 752, 148 
Osterbrock D. E., Ferland G. J., 2006, Astrophysics of Gas Nebulae and Active Galactic Nuclei. University Science Books, Mill Valley, CA

Parsa S., Dunlop J. S., McLure R. J., 2017, MNRAS, 474, 2904

Pei Y. C., 1992, ApJ, 395, 130

Pérez-Montero E., Amorín R., 2017, MNRAS, 467, 1287

Planck Collaboration et al., 2016, A\&A, 596, A108

Rasappu N., Smit R., Labbé I., Bouwens R. J., Stark D. P., Ellis R. S., Oesch P. A., 2016, MNRAS, 461, 3886

Robertson B. E. et al., 2013, ApJ, 768, 71

Robertson B. E., Ellis R. S., Furlanetto S. R., Dunlop J. S., 2015, ApJ, 802, L19

Schaerer D., Izotov Y. I., Verhamme A., Orlitová I., Thuan T. X., Worseck G., Guseva N. G., 2016, A\&A, 591, L8

Schlafly E. F., Finkbeiner D. P., 2011, ApJ, 737, 103

Schmidt K. B. et al., 2017, ApJ, 839, 17

Senchyna P. et al., 2017, MNRAS, 472, 2608

Shim H., Chary R.-R., Dickinson M., Lin L., Spinrad H., Stern D., Yan C.-H., 2011, ApJ, 738, 69

Shirazi M., Brinchmann J., 2012, MNRAS, 421, 1043

Shivaei I. et al., 2018, ApJ, 855, 42

Skilling J. et al., 2006, Bayesian Anal., 1, 833

Smit R. et al., 2014, ApJ, 784, 58

Smit R., Bouwens R. J., Labbé I., Franx M., Wilkins S. M., Oesch P. A., 2016, ApJ, 833, 254

Smit R., Swinbank A. M., Massey R., Richard J., Smail I., Kneib J.-P., 2017, MNRAS, 467, 3306

Stark D. P. et al., 2015a, MNRAS, 450, 1846

Stark D. P. et al., 2015b, MNRAS, 454, 1393

Stark D. P. et al., 2017, MNRAS, 464, 469

Stark D. P., 2016, ARA\&A, 54, 761

Stark D. P., Schenker M. A., Ellis R., Robertson B., McLure R., Dunlop J., 2013, ApJ, 763, 129

Stasińska G., Izotov Y., Morisset C., Guseva N., 2015, A\&A, 576, A83
Strom A. L., Steidel C. C., Rudie G. C., Trainor R. F., Pettini M., Reddy N. A., 2017, ApJ, 836, 164

Trebitsch M., Blaizot J., Rosdahl J., Devriendt J., Slyz A., 2017, MNRAS, 470,224

Vale Asari N., Stasińska G., Morisset C., Cid Fernandes R., 2016, MNRAS, 460, 1739

Vanzella E. et al., 2016, ApJ, 825, 41

Verhamme A., Orlitová I., Schaerer D., Hayes M., 2015, A\&A, 578, A7

Verhamme A., Orlitová I., Schaerer D., Izotov Y., Worseck G., Thuan T. X., Guseva N., 2017, A\&A, 597, A13

Vidal-García A., Charlot S., Bruzual G., Hubeny I., 2017, MNRAS, 470, 3532

Wise J. H., Cen R., 2009, ApJ, 693, 984

Wise J. H., Demchenko V. G., Halicek M. T., Norman M. L., Turk M. J., Abel T., Smith B. D., 2014, MNRAS, 442, 2560

Zackrisson E., Inoue A. K., Jensen H., 2013, ApJ, 777, 39

\section{SUPPORTING INFORMATION}

Supplementary data are available at MNRAS online.

Table 1. UV and optical emission lines fluxes computed from the $H S T / C O S$, MMT, and sDss spectra, along with the fluxes predicted by our model.

Please note: Oxford University Press is not responsible for the content or functionality of any supporting materials supplied by the authors. Any queries (other than missing material) should be directed to the corresponding author for the article.

This paper has been typeset from a $\mathrm{T}_{\mathrm{E}} \mathrm{X} / \mathrm{L} \mathrm{T} \mathrm{E} \mathrm{X}$ file prepared by the author. 